\title{
THE EFFECTIVE ACTION IN GAUGED SUPERGRAVITY ON HYPERBOLIC BACKGROUND AND INDUCED COSMOLOGICAL CONSTANT
}

\author{
A.A. Bytsenko \\ Department of Theoretical Physics, State Technical University, \\ St Petersburg 195251, Russia \\ S. D. Odintsov円 \\ Department E.C.M., Faculty of Physics, University of Barcelona, \\ Diagonal 647, 08028 Barcelona, Spain \\ and Pedagogical Institute, 634041, Tomsk, Russia \\ S. ZERBINI 2 \\ Department of Physics, University of Trento, 38050 Povo, Italy \\ and I.N.F.N., Gruppo Collegato di Trento
}

\begin{abstract}
The one-loop effective action for 4-dimensional gauged supergravity with negative cosmological constant, is investigated in space-times with compact hyperbolic spatial section. The explicit expansion of the effective action as a power series of the curvature on hyperbolic background is derived, making use of heat-kernel and zeta-regularization techniques. The induced cosmological and Newton constants are computed.
\end{abstract}

\section{PACS number(s): 04.60 Quantum theory of gravitation}

One of the basic motivations for studying the quantum gravity on de Sitter background 过, 2, 3, 4, 5, [6, 7, 8, 10, 11, 9, 12] has been the fact that it might be a very reasonable candidate for vacuum state when the classical action contains a positive cosmological term. It has also been expected that such investigations might also provide the framework to solve the cosmological constant problem [1, 2, 1, 5, 11]. In particular, in Ref. [12] a quite interesting model of Coleman-Weinberg type suppression for the effective cosmological constant (due to the radiative corrections of Einstein gravity) has been suggested. One important ingredient of this model has been the proposal to study the large-distance limit of the quantum gravity one-loop effective action (for a general review on effective action, see for example [13]) on the de Sitter background. However, the use of such background has been shown to be inadeguate to implement the cosmological constant suppression mechanism. With regard to this issue, the use of an hyperbolic background (i.e. a gravitational theory with negative cosmological constant) might slighty improve the situation, as it has been recently shown in Ref. [14], where the pure gravity has been treated as an example.

There exists a class of theories (gauged supergravities) for which hyperbolic space-times are the natural vacuum states at, since for these models the effective cosmological constant classically may often be negative. It is the purpose of this work to investigate, in the large-distance limit, the Euclidean one-loop effective action for the $O(4)$ gauged supergravity on a hyperbolic background of the form $H^{4} / \Gamma$ (i.e. compact hyperbolic 4-dimensional manifold), making use of heat-kernel, Selberg trace formula and zeta-function regularization techniques [15, 16, 17]. The possibility to realize the cosmological constant suppression mechanism in the spirit of Ref. [12] is investigated and it is shown that some impletmentation of it may be achieved.

\footnotetext{
${ }^{1}$ E-mail address: odintsov@ebubecm1.bitnet

${ }^{2}$ E-mail address: zerbini@science.unitn.it
} 
To start with, let us briefly recall the general formalism enabling the treat the one-loop effective action. In general, the background geometry may be chosen to correspond to constant curvature space: Euclidean space $\mathbb{R}^{4}$ with constant curvature $k=0$, the sphere $S^{4}$ of radius $a\left(k=a^{-2}\right)$ and the hyperbolic space $H^{4}\left(k=-a^{-2}\right)$. For all of these spaces, the curvature tensor, the Ricci tensor and the scalar curvature have respectively the form

$$
\begin{aligned}
R_{\alpha \beta \gamma \delta} & =k\left(g_{\alpha \gamma} g_{\beta \delta}-g_{\alpha \delta} g_{\beta \gamma}\right), \\
R_{\alpha \beta} & =k(N-1) g_{\alpha \beta}, \quad R=k N(N-1) .
\end{aligned}
$$

Here we shall mainly deal with the (compact) hyperbolic case. After a standard functional integration the Euclidean one-loop effective action may be written

$$
\Gamma^{(1)}=\frac{1}{2} \sum_{p, i} C_{p} \log \operatorname{det}\left(O_{p}^{(i)} / \mu^{2}\right),
$$

where $\mu^{2}$ is a normalization parameter, $i=0,1 / 2,1,3 / 2,2$ refer to scalars, $\ldots$. transverse symmetric traceless second rank tensors respectively and $C_{p}$ are the weights associated with the Laplace-type operators $O_{p}^{(i)}$. However, it is well known that, in the path-integral formulation of Euclidean quantum gravity, some of these operators are negative. In the following, we shall assume that, when necessary, the contour rotations and field redefinitions, in accordance with the prescriptions of Refs. [1, 19], have been done.

In this paper we shall mainly consider $\Lambda<0$ and the determinants of the operators $O_{p}^{(i)}$ will be regularized by means of the zeta-function technique [15]. The fact we are dealing with the case $\Lambda<0$, implies that all $O_{p}^{(i)}$ (provided the integration over imaginary field is performed when necessary) are, for $a$ sufficiently large, positive definite. Thus, a generic Laplace-type operator can be written as

$$
O_{p}^{(i)}=a^{-2} L_{p}^{(i)}
$$

where we have introduced the dimensionless operator $L_{p}^{(i)}$ of the form

$$
L_{p}^{(i)}=L^{(i)}+X_{p}^{(i)}, \quad X_{p}^{(i)}=a^{2}\left|\Lambda_{p}^{(i)}\right|+\nu_{p}^{(i)},
$$

where $L=-\nabla_{N}^{2}$ is the Laplace-Beltrami operator on $M^{4}, \Lambda_{p}^{(i)}=b_{p}^{(i)} \Lambda, b_{p}^{(i)}$ is a non-negative number, $\nu_{p}^{(i)}$ are known constants and we assume $X_{p}^{(i)}>0$. The zeta-function regularization gives

$$
\log \operatorname{det}\left(O_{p}^{(i)} / \mu^{2}\right)=-\left[\zeta^{\prime}\left(0 \mid O_{p}^{(i)}\right)+\log \mu^{2} \zeta\left(0 \mid O_{p}^{(i)}\right)\right],
$$

where

$$
\begin{aligned}
\zeta\left(s \mid O_{p}^{(i)}\right) & =\frac{1}{\Gamma(s)} \int_{0}^{\infty} d t t^{s-1} \operatorname{Tr} e^{-t O_{p}^{(i)}} \\
& =a^{2 s} \zeta\left(s \mid L_{p}^{(i)}\right) .
\end{aligned}
$$

Now we have

$$
\zeta\left(z \mid L_{p}^{(i)}\right)=\frac{1}{\Gamma(z)} \int_{0}^{\infty} d t t^{z-1} e^{-t X_{p}^{(i)}} \operatorname{Tr} e^{-t L}
$$

On general grounds, one can write the well known heat-kernel expansion, valid for $t \rightarrow 0$

$$
\operatorname{Tr} e^{-t L} \simeq \sum_{r} K_{r} t^{\frac{r-4}{2}},
$$


where the coefficients $K_{r}$ associated with the operator $L$ can be, in principle, computed and depend on the geometry of the compact manifold. The expansion (8) is valid for a compact smooth manifold with or without boundary. We shall consider manifolds without boundary, which a possible presence of conical singularities. For boundaryless manifolds $K_{r}=0$ when $r$ is odd.

If we make use of the general heat-kernel expansion, we get

$$
\zeta\left(s \mid O_{p}^{(i)}\right)=\frac{a^{2 s}}{\Gamma(s)}\left\{\sum_{r} K_{2 r} \Gamma(s+r-2) X_{p}^{2-s-r}+J_{p}(s)^{(i)}\right\} .
$$

where $J_{p}(s)^{(i)}$ are entire functions of $s$. The first gamma functions have a simple pole at $s=0$. So we have

$$
\zeta\left(0 \mid O_{p}^{(i)}\right)=\sum_{l=0}^{2} K_{4-2 l}^{(i)} \frac{\left(-X_{p}^{(i)}\right)^{l}}{l !}
$$

and

$$
\begin{aligned}
\zeta^{\prime}\left(0 \mid O_{p}^{(i)}\right) & =\sum_{l=0}^{2} K_{4-2 l}^{(i)} \frac{\left(-X_{p}^{(i)}\right)^{l}}{l !}\left(\gamma+\Psi(l+1)+\log \left(a^{2} / X_{p}^{(i)}\right)\right) \\
& +\sum_{r>2} K_{2 r}^{(i)} \Gamma(r-2)\left(X_{p}^{(i)}\right)^{2-r}+J_{p}^{(i)}(0) .
\end{aligned}
$$

As a result, the one-loop contribution to the effective action reads

$$
\begin{aligned}
\Gamma^{(1)} & =-\frac{1}{2} \sum_{p, i} C_{p}\left\{\log \left(a^{2} \mu^{2} / X_{p}^{(i)}\right) \zeta\left(0 \mid O_{p}^{(i)}\right)+\sum_{l=0}^{2} K_{4-2 l} \frac{\left(-X_{p}^{(i)}\right)^{l}}{l !} F(l)\right. \\
& \left.+\sum_{r>2} K_{2 r}^{(i)} \Gamma(r-2)\left(X_{p}^{(i)}\right)^{2-r}+J_{p}^{(i)}(0)\right\},
\end{aligned}
$$

where $F(0)=0$ and $F(l)=\sum_{j=1}^{l} j^{-1}$.

Up to now, our results have a general form. However, in order to perform explicit computations the knowledge of the heat-kernel coefficients $K_{2 r}$ and the analytical part $J_{p}(s)$ are necessary. It is well known that the $K_{2 r}$ coefficients are, in principle, computable. On the other hand, the evaluation of the $J_{p}(s)$ functions requires an analytical continuation and this is achieved, usually, throught the explicit knowledge of the spectrum of the operator $L_{p}$. Unfortunately on hyperbolic backgrounds (the case under discussion) the spectrum of the Laplace operator is not explicitly known. Moreover, the scalar sector may be investigated by making use of Selberg trace formula techniques. For higher spin fields, the analogue techniques is more complicated and, to our knowledge, it is not explicitly known. In such situation, in order to perform explicit calculations, we have to make use of a further approximation scheme, namely the large-distance limit, which is particulary interesting in pure gravity and it has been proposed in Ref. [12] for spherical 4-dimensional background in the discussion of the cosmological constant issue.

In our language, the large distance limit is equivalent to find the asymptotics of the effective action for very large $X_{p}$ (note that $b_{p}$ must be non-vanishing and we left understood that, in the sum over $p$, the terms corresponding to some $b_{p}=0$, must be omitted). Thus, in this limit we can neglect the terms related to $r>2$ and the analytical (normally unknown) terms. Thus, retaining only the leading terms

$$
\zeta\left(0 \mid O_{p}^{(i)}\right)=\frac{1}{2} a^{4}\left|\Lambda_{p}^{(i)}\right|^{2} K_{0}^{(i)}+a^{2}\left|\Lambda_{p}^{(i)}\right| 2\left(\nu_{p} K_{0}^{(i)}-K_{2}^{(i)}\right)+\ldots .
$$




$$
\begin{aligned}
\zeta^{\prime}\left(0 \mid O_{p}^{(i)}\right) & =\frac{1}{2}\left\{a^{4}\left|\Lambda_{p}^{(i)}\right|^{2}\left(-\log \left|\Lambda_{p}^{(i)}\right|+F(2)\right) K_{0}^{(i)}\right. \\
& +a^{2}\left|\Lambda_{p}^{(i)}\right|\left[-2 \log \left|\Lambda_{p}^{(i)}\right|\left(\nu_{p}^{(i)} K_{0}^{(i)}-K_{2}^{(i)}\right)\right. \\
& \left.\left.+\nu_{p}^{(i)} K_{0}^{(i)}(2 F(2)-1)-2 F(1) K_{2}^{(i)}\right]+\ldots\right\} .
\end{aligned}
$$

Let us introduce a physical scale by means of the following redefinition of the $\mu^{2}$ parameter

$$
\log \frac{\mu^{2}}{\left|\Lambda_{p}\right|}+F(2) \mapsto-\log \left(|\Lambda| \mu^{-2}\right)
$$

Hence, the leading part of $\Gamma^{(1)}$ is given by

$$
\begin{aligned}
\Gamma^{(1)} & =\frac{\log \left(|\Lambda| \mu^{-2}\right)}{4} \sum_{p, i} C_{p}^{(i)}\left\{a^{4}\left|\Lambda_{p}^{(i)}\right|^{2} K_{0}^{(i)}\right. \\
& \left.+a^{2}\left|\Lambda_{p}^{(i)}\right|\left(\nu_{p}^{(i)} K_{0}^{(i)}-K_{2}^{(i)}\right)+\ldots\right\} .
\end{aligned}
$$

Now it is quite easy to rewrite the above one-loop corrections in terms of geometric quantities, appearing in the classical action. To this aim, it is sufficient to observe that, for constant curvature space, we have

$$
\begin{aligned}
a^{4} & =\int d^{4} x \sqrt{g}\left(\operatorname{Vol}\left(\mathcal{F}_{N}\right)\right)^{-1}, \\
a^{2} & =\int d^{4} x \sqrt{g} \kappa R\left(12 \operatorname{Vol}\left(\mathcal{F}_{N}\right)\right)^{-1},
\end{aligned}
$$

where $\kappa=k a^{2}, \mathcal{F}_{N}$ is the fundamental domain of the compact hyperbolic manifold

As a result, using the relevant part of $\Gamma^{(1)}$ and the classical action, one can write the one-loop effective action in the large-distance limit as

$$
\begin{aligned}
\Gamma_{e f f} & =S+\Gamma^{(1)}=\int d^{4} x \sqrt{g}\left[\Lambda(8 \pi G)^{-1}+\beta_{\Lambda}|\Lambda|^{2} \log \left(|\Lambda| \mu^{-2}\right)\right] \\
& -\int d^{4} x \sqrt{g} R\left[(16 \pi G)^{-1}+\beta_{G}|\Lambda| \log \left(|\Lambda| \mu^{-2}\right)\right]
\end{aligned}
$$

where

$$
\begin{aligned}
& \beta_{\Lambda}=\frac{1}{4 V\left(\mathcal{F}_{N}\right)} \sum_{p, i}\left(b_{p}^{(i)}\right)^{2} C_{p}^{(i)} K_{0}^{(i)}, \\
& \beta_{G}=-\frac{\kappa}{24 V\left(\mathcal{F}_{N}\right)} \sum_{p, i} b_{p}^{(i)} C_{p}^{(i)}\left(\nu_{p}^{(i)} K_{0}^{(i)}-K_{2}^{(i)}\right) .
\end{aligned}
$$

The effective Newton and cosmological constants turn out to be

$$
\begin{aligned}
& \Lambda_{e f f}=\Lambda \frac{1+\kappa \beta_{\Lambda} 8 \pi G|\Lambda| \log \left(|\Lambda| \mu^{-2}\right)}{1+\beta_{G} 16 \pi G|\Lambda| \log \left(|\Lambda| \mu^{-2}\right)}, \\
& (G \Lambda)_{e f f}=(G \Lambda) \frac{1+\kappa \beta_{\Lambda} 8 \pi G|\Lambda| \log \left(|\Lambda| \mu^{-2}\right)}{\left[1+\beta_{G} 16 \pi G|\Lambda| \log \left(|\Lambda| \mu^{-2}\right)\right]^{2}} .
\end{aligned}
$$

In order to apply the general formula, we have to compute the Seeley-deWitt cofficients $K_{2 r}^{(i)}$, associated with the (constrained) spin-fields on an compact constant curvature background, and 
this could be done making use of the general algorithm. In the case of compact hyperbolic background $H^{4} / \Gamma$, we may proceed as following.

For scalar (spinor) fields, if we limit ourselves to a smooth manifolds (strictly hyperbolic subgroup of $\Gamma$ ), one can use the Selberg trace formula. In the case of scalar fields we have (see [18] for details)

$$
\begin{aligned}
\operatorname{Tr} e^{-t L^{(0)}} & =V\left(\mathcal{F}_{4}\right) \int_{-\infty}^{\infty} \frac{r\left(r^{2}+\frac{1}{4}\right) \tanh \pi r}{8 \pi^{2}} e^{-\left(r^{2}+\frac{9}{4}\right) t} d r \\
& +\sum_{\{\gamma\}} \sum_{n=1}^{\infty} \frac{\chi(\gamma)^{n} l_{\gamma}}{S_{4}\left(n, l_{\gamma}\right)} \frac{1}{\sqrt{4 \pi t}} \exp \left[-\left(\frac{9}{4} t+\frac{n^{2} l_{\gamma}^{2}}{4 t}\right)\right] .
\end{aligned}
$$

Above we have the first term (identity contribution) and the topological one, wich however is exponentially small for $t \rightarrow 0$. Thus the heat kernel coefficients are contained in the identity term, which are the contribition related to $H^{4}$, apart the volume normalization. This means that the contribution which are interested in are computable, for higher spins, from the exact heat-kernel for the Laplace type operator on $H^{4}$. This heat-kernel can be computed using the result on the constrained higher-spin Plancherel measure $\mu^{(s)}(r)$ on $H^{4}$, evaluated in Ref.[20]. The heat-kernel trace can be written as

$$
K\left(t \mid L^{(s)}\right)=V\left(\mathcal{F}_{4}\right) e^{-t\left(\frac{9}{4}+s\right)} \int_{0}^{\infty} e^{-t r^{2}} \mu^{(s)}(r) d r
$$

where

$$
\mu^{(s)}(r)=\frac{(2 s+1)}{8 \pi^{2}}\left[\left(r^{2}+\left(s+\frac{1}{2}\right)^{2}\right] \tanh \pi(r+i s) .\right.
$$

Making use of

$$
\tanh \pi(r+i s)=\left\{\begin{array}{l}
1-\frac{2}{1+e^{2 \pi r}} \quad, \quad s=0,1,2, \ldots \\
1-\frac{2}{1-e^{2 \pi r}} \quad, \quad s=1 / 2,3 / 2, \ldots
\end{array}\right.
$$

and the identities

$$
\begin{aligned}
\int_{0}^{\infty} \frac{r^{2 n-1}}{e^{2 \pi r}+1} d r & =\frac{(-1)^{n-1}\left(1-2^{1-2 n}\right)}{4 n} B_{2 n} \\
\int_{0}^{\infty} \frac{r^{2 n-1}}{e^{2 \pi r}-1} d r & =\frac{(-1)^{n-1}}{4 n} B_{2 n}
\end{aligned}
$$

in which $B_{2 n}$ are the Bernoulli numbers, one obtains the first Seeley-DeWitt coefficients related to spin-constrained Laplacian $L^{(s)}$ :

For $s=0,1,2,$. .

$$
\begin{aligned}
& K_{0}^{(s)}=\frac{(2 s+1)}{(4 \pi)^{2}} V\left(\mathcal{F}_{4}\right) \\
& K_{2}^{(s)}=\frac{(2 s+1)}{(4 \pi)^{2}} V\left(\mathcal{F}_{4}\right)\left(s^{2}-2\right)=\left(s^{2}-2\right)(2 s+1) K_{0}^{(0)} \\
& K_{4}^{(s)}=\frac{(2 s+1)}{(4 \pi)^{2}} V\left(\mathcal{F}_{4}\right)\left[\left(\frac{9}{4}+s\right)\left(\frac{7}{8}-s^{2}-\frac{s}{2}\right)-\frac{1}{12}\left(s+\frac{1}{2}\right)^{2}+\frac{7}{40}\right]
\end{aligned}
$$

For $s=1 / 2,3 / 2, \ldots$

$$
\begin{aligned}
& K_{0}^{(s)}=\frac{(2 s+1)}{(4 \pi)^{2}} V\left(\mathcal{F}_{4}\right) \\
& K_{2}^{(s)}=\frac{(2 s+1)}{(4 \pi)^{2}} V\left(\mathcal{F}_{4}\right)\left(s^{2}-2\right)=\left(s^{2}-2\right)(2 s+1) K_{0}^{(0)} \\
& K_{4}^{(s)}=\frac{(2 s+1)}{(4 \pi)^{2}} V\left(\mathcal{F}_{4}\right)\left[\left(\frac{9}{4}+s\right)^{2}-\left(\frac{9}{2}+2 s-\frac{1}{3}\right)\left(s+\frac{1}{2}\right)^{2}+\frac{1}{30}\right]
\end{aligned}
$$


From the above equations, we obtain

$$
\begin{aligned}
& \beta_{\Lambda}=\frac{1}{4 V\left(\mathcal{F}_{4}\right)} \sum_{p, i}\left(b_{p}^{(i)}\right)^{2} C_{p}^{(i)} K_{0}^{(i)}==\frac{1}{4(4 \pi)^{2}} \sum_{p, i}(2 i+1)\left(b_{p}^{(i)}\right)^{2} C_{p}^{(i)}, \\
& \beta_{G}=\frac{1}{24 V\left(\mathcal{F}_{4}\right)} \sum_{p, i} b_{p}^{(i)} C_{p}^{(i)}\left(\nu_{p} K_{0}^{(i)}-K_{2}^{(i)}\right)=\frac{1}{(24 \pi)^{2}} \sum_{p, i} b_{p}^{(i)} C_{p}^{(i)}(2 i+1)\left(\nu_{p}^{(i)}-i^{2}+2\right),
\end{aligned}
$$

The main interest in using a supergravity theory stems from the fact that the bosonic and fermionic degrees of freedom enter the one-loop path integral with opposite contributions $\left(C_{p}^{(s)}\right.$ have opposite signs). Thus, there might be the possibility to have a cancellation in the $\beta_{\Lambda}$ expression, which is impossible in a pure gravity theory [14. This is also supported by the improved one-loop ultraviolet behaviour related to every supergravity theory.

Our next aim will be to illustrate these general considerations to the $\mathrm{O}(4)$ gauged supergravity. The Lagrangian for this theory is given by [21, 11]

$$
\begin{aligned}
L & =-\frac{R}{K}+\frac{2}{K^{2}} \frac{\partial_{\mu} \Phi \partial^{\mu} \Phi^{*}}{\left(1-|\Phi|^{2}\right)^{2}}-\frac{8 g^{2}}{K^{4}}\left(1+\frac{2}{1-|\Phi|^{2}}\right) \\
& +\frac{1}{8}\left(F_{\mu \nu}^{i j}\right)^{2}+\left\{\frac{1}{8} \frac{\Phi}{1-|\Phi|^{2}}\left(\Phi \delta_{i k} \delta_{j l}-\frac{1}{2} \epsilon_{i j k l}\right) F_{\mu \nu}^{i j}\left(F_{\mu \nu}^{k l}+i \tilde{F}_{\mu \nu}^{k l}\right)+h . c .\right. \\
& +\frac{1}{2} \epsilon^{\mu \nu \rho \sigma} \bar{\Psi}_{\mu}^{i} \gamma_{5} \gamma_{\nu} D_{\rho} \Psi_{\sigma}^{i}+\frac{2 g}{K \sqrt{\left(1-|\Phi|^{2}\right)}} \bar{\Psi}_{\mu}^{i} \sigma_{\mu \nu} \Psi_{\nu}^{i} \\
& +\frac{1}{2} \bar{\chi}^{i} \gamma_{\mu} D_{\mu} \chi^{i}+\frac{\sqrt{2} g}{K \sqrt{\left(1-|\Phi|^{2}\right)}} \bar{\Psi}_{\mu}^{i} \gamma_{\mu}\left(\Phi_{1}+i \gamma_{5} \Phi_{2}\right) \chi^{i} \\
& + \text { (quartic fermionic terms), }
\end{aligned}
$$

where $K^{2}=16 \pi G, \sigma_{\mu \nu}=\frac{1}{2}\left[\gamma_{\mu}, \gamma_{\nu}\right],\left\{\gamma_{\mu}, \gamma_{\nu}\right\}=g_{\mu \nu}, \gamma_{5}^{2}=1, \tilde{F_{\mu \nu}}=\frac{1}{2} \epsilon^{\mu \nu \rho \sigma} F_{\rho \sigma}$ and $D_{\mu}$ is the total covariant gravitational and gauge derivative. The physical content of the fields are: the gravitons, $A_{\mu}^{i} O(4)$ gauge fields $(i, j=1, \ldots 4)\left(F_{\mu \nu}^{k l}\right.$ being the field strenght), a complex scalar field $\Phi=\Phi_{1}+i \Phi_{2}$, satisfying the constraint $|\Phi|<1$, four $\Psi_{\mu}^{i}$ (Majorana gravitinos) and four $\chi^{i}$ (Majorana spinors). The related action is invariant under gauged $N=4$ supersymmetry (for details see [21, 22, 11]). We will make the one loop-approximation expanding all the fields around the stable supersymmetric vacuum [1]

$$
\Phi=0, \Psi=0, A=0, \chi=0, \Lambda_{\text {on shell }}=\Lambda_{0}=-\frac{12 g^{2}}{K^{2}}
$$

thus $g_{\mu \nu}$ correspond to anti-de Sitter space and the tree-level cosmological constant turns out to be negative, namely

$$
\Lambda=-\frac{g^{2}}{K^{2}}(1+2 \alpha) \quad \alpha=\left(1-|\Phi|^{2}\right)^{-1}
$$

Note that $\Phi=0$ is the only extremum (maximum) of the classical potential (third term in Eq. (32)).

The one-loop Euclidean effective action on the background (33) has been evaluated (making use of the De Donder gauge for the graviton field and $\Psi=0$ gauge for the gravitino) in Ref. [11]. The result reads

$$
\begin{aligned}
\Gamma^{(1)} & =\frac{1}{2} \log \operatorname{det}\left(-\nabla_{2}^{2}+2|\Lambda|-\frac{8}{a^{2}}\right)-\frac{1}{2} \log \operatorname{det}\left(-\nabla_{1}^{2}+\frac{3}{a^{2}}\right)+3 \log \operatorname{det}\left(-\nabla_{1}^{2}-\frac{3}{a^{2}}\right) \\
& +\frac{1}{2} \log \operatorname{det}\left(-\nabla_{0}^{2}-4|\Lambda|+\frac{12}{a^{2}}\right)-\frac{7}{2} \log \operatorname{det}\left(-\nabla_{0}^{2}\right)+\frac{1}{2} \log \operatorname{det}\left(-\nabla_{0}^{2}-2|\Lambda| \frac{4 \alpha-3}{1+2 \alpha}\right)
\end{aligned}
$$




$$
\begin{aligned}
& +\frac{1}{2} \log \operatorname{det}\left(-\nabla_{0}^{2}-2|\Lambda| \frac{1}{1+2 \alpha}\right)-\log \operatorname{det}\left(-\nabla_{3 / 2}^{2}+|\Lambda| \frac{\alpha}{1+2 \alpha}\right) \\
& +\log \operatorname{det}\left(-\nabla_{1 / 2}^{2}+4|\Lambda| \frac{\alpha}{1+2 \alpha}\right)-\log \operatorname{det}\left(-\nabla_{1 / 2}^{2}\right),
\end{aligned}
$$

in which the $0,1 / 2,1,3 / 2,2$ are labelling the scalar, spinor, tranverse vector, transverse gravitino and traceless transverse tensor respectively.

In our approach, all the $O_{p}^{(i)}$ operators should be positive definite. One can show that in the scalar sector, some operators are negative definite. Furthermore, the indefiniteness may depend on the gauge fixing. There exist several possibilities to handle this problem. One of these might consist in considering a suitable analytical continuation, when necessary, from $-|\Lambda|$ to $|\Lambda|$. This regularization procedure gives finite results, but the price to pay is the appearance of imaginary terms in the effective action, signalling a quantum metastability. However, in the large-distance limit, we may formally consider some $b_{p}^{(s)}<0$, without any problem, the imaginary terms being non leading.

Keeping in mind such observations, Eq. (35) gives

$$
\beta_{\Lambda}=\frac{1}{(4 \pi)^{2}} \frac{66 \alpha^{2}+12 \alpha+19}{(1+2 \alpha)^{2}}
$$

and

$$
\beta_{G}=-\frac{1}{24(4 \pi)^{2}} \frac{185 \alpha+76}{(1+2 \alpha)}
$$

which are an example of computation.

Finally we would like briefly to comment on the issue related to richer geometric structure we are dealing with. In the explicit 4-dimensional example presented for illustrative purposes, we have considered a smooth compact hyperbolic manifold $H^{4} / \Gamma$. Technically this is equivalent to the assumption that only hyperbolic elements are present. However, it is known that also elliptic elements may be present, and their contribution can be taken into account by means of the Selberg trace formula. It is expected an elliptic correction to $\beta_{G}$ of the form 14]

$$
\beta_{G}=-\frac{1}{24(4 \pi)^{2}}\left[\frac{185 \alpha+76}{(1+2 \alpha)}+\sum_{p, i} b_{p}^{(i)} C_{p}^{(i)} \frac{K_{2, E}^{(i)}}{K_{0}^{(0)}}\right]
$$

where $K_{2, E}^{(i)}$ are the contributions related to the elliptic elements of $\Gamma$.

In conclusion, we have discussed the one-loop effective action for $O(4)$ supergravity with negative cosmological constant on a hyperbolic background, by making use of zeta-function regularization and heat-kernel techniques. The use of large-distant limit approximation has permitted to obtain reasonable simple expressions for the effective one-loop cosmological and gravitational constants. These expressions, in the large-distance limit, depend on the heat-kernel coefficients, which can be computed. A novel feature, with respect to the spherical $(\Lambda>0)$ background, consists in the richer geometric structure one has to deal with. As a consequence, the value of the coefficient $\beta_{G}$ may also depends on the choice of the topological non-trivial field configurations (twisted or untwisted fields, see also [23] for discussion of twisted fields on toroidal spaces) on the orbifolds $H^{4} / \Gamma$. 


\section{Acknowledgments}

We thank G. Cognola and L. Vanzo for discussions. A.A. Bytsenko wishes to thank INFN and the Department of Physics of Trento University for financial support and kind hospitality. S.D. Odintsov thanks the Generalitat de Catalonya for financial support.

\section{References}

[1] G.W. Gibbons, S.W. Hawking and M.J. Perry. Nucl. Phys., B138, 141, (1978).

[2] G.W. Gibbons and M.J. Perry. Nucl. Phys.,B146, 90, (1978).

[3] S.W. Hawking. The path-integral approach to quantum gravity. In General Relativity. An Einstein Centenary Survey, S.W. Hawking and W. Israel, editors. Cambridge University Press, Cambridge, (1979).

[4] S.M. Christensen and M.J. Duff. Nucl. Phys., B170, 480, (1980).

[5] S.M. Christensen, M.J. Duff, G.W. Gibbons and M. Rocek. Phys. Rev. Lett., 45, 161, (1980).

[6] I. Antoniadis, J. Iliopulos and T.N. Tomaras. Phys. Rev. Lett., 56, 1319, (1986).

[7] L. Ford. Phys. Rev., D31, 710 (1985).

[8] B. Allen and M. Turyn. Nucl. Phys., B292, 813, (1987).

[9] S. D. Odintsov. Europhys. Lett., 10, 287, (1989); Theor. Math. Phys., 82, 61, (1990).

[10] I. Antoniadis and E. Mottola. J. Math. Phys., 32, 1037, (1991).

[11] E.S. Fradkin and A.A. Tseytlin. Nucl. Phys., B234, 472, (1984).

[12] T.R. Taylor and G. Veneziano. Nucl. Phys., B345, 210, (1990).

[13] I.L. Buchbinder, S.D. Odintsov and I.L. Shapiro. Effective action in quantum gravity. IOP Publishing, Bristol and Philadelphia, (1992).

[14] A.A. Bytsenko, S.D. Odintsov and and S. Zerbini. Trento University preprint UTF 328, (1994).

[15] S.W. Hawking. Commun. Math. Phys., 55, 133, (1977).

[16] J. Dowker and R.Critchley. Phys. Rev. D13, 3224,(1976).

[17] E. Elizalde, S.D. Odintsov, A. Romeo, A.A. Bytsenko and S. Zerbini. Zeta-regularization with applications.World Sci., Singapore, 1994.

[18] A.A. Bytsenko, G. Cognola, L. Vanzo and S. Zerbini. Quantum fields and extended objects in space-times with constant curvature spatial section. Trento University Preprint UTF 325, (1994).

[19] J. Polchinski Phys. Lett., B219, 251, (1989).

[20] R. Camporesi and A. Higuchi. Phys. Rev., D47, 3339, (1993). 
[21] A. Das,M. Fisler and M. Rocek. Phys. Rev., D16, 3427, (1977); B. de Wit and H. Nicolai. Nucl. Phys., B188,98, (1981);Nucl. Phys., B208,323, (1982)

[22] P. Breitenlohner and D. Z. Freedman. Ann.. Phys., 144, 249, (1982); G.W. Gibbons, C. M. Hull and N.P. Warner Nucl. Phys., B218, 173, (1983).

[23] S.J.Avis and C.J.Isham. Nucl. Phys.,B156, 441, (1979). 